\title{
Etude R.P.E. des déformations plastiques dans le corindon
}

\author{
B. Champagnon, E. Duval, B. Tribollet \\ Laboratoire de Spectroscopie des Solides, Université Lyon I, 69622 Villeurbanne Cedex 1, France
}

et J. L. Cadoz

Laboratoire de Physique des Matériaux, 92190 Meudon, France

(Reçu le 23 juillet 1982, révisé le 11 octobre, accepté le 4 novembre 1982)

\begin{abstract}
Résumé. - Les mesures de R.P.E. et de spectroscopie optique ont été effectuées sur des échantillons de rubis $\left(\mathrm{Al}_{2} \mathrm{O}_{3}: \mathrm{Cr}^{3+}\right)$ déformés suivant les systèmes de glissement basal et prismatique. Dans le cas d'un glissement basal, certaines raies de R.P.E. sont scindées, ce qui, en liaison avec les résultats optiques, permet d'obtenir des résultats quantitatifs sur la désorientation due aux déformations plastiques.
\end{abstract}

\begin{abstract}
E.P.R. and optic measurements have been carried out on ruby $\left(\mathrm{Al}_{2} \mathrm{O}_{3}: \mathrm{Cr}^{3+}\right)$ crystals deformed by compressions along the basal and prismatic glide planes. For a basal slip splittings of E.P.R. lines are observed; by connexion with optical measurements quantitative results on the disorientation due to plastic deformations are obtained.
\end{abstract}

Dans cette étude, nous nous proposons de déterminer les effets des déformations plastiques sur le corindon $\left(\alpha-\mathrm{Al}_{2} \mathrm{O}_{3}\right)$. Les méthodes utilisées, Résonance Paramagnétique Electronique (R.P.E.) ou Optique, ne peuvent s'appliquer que si le corindon est dopé par une faible quantité d'un ion paramagnétique possédant un spectre optique dans le visible. Nous avons choisi l'ion $\mathrm{Cr}^{3+}$ dont la spectroscopie R.P.E. [1-4], la spectroscopie optique $[5,6]$ ainsi que l'influence des contraintes sur ces spectres [7-11] sont bien connues. Le dopage est suffisamment faible $(0,1$ atome $\%)$ pour que les impuretés n'aient pas une influence déterminante sur la plasticité des cristaux.

La R.P.E. est largement utilisée pour étudier l'influence soit de contraintes uniaxiales soit de contraintes distribuées au hasard autour d'un ion paramagnétique $[12,13]$. Son utilisation pour l'étude des déformations plastiques est plus récente $[14,15]$. L'intérêt de la R.P.E. par rapport aux méthodes d'observation telles que la microscopie électronique est qu'elle permet d'observer en volume et non pas dans une lame mince les différents défauts dus aux déformations plastiques.

Dans ce travail, nous avons étudié les effets de glissements dus à différents types de déformation plastique. Nous avons mis en évidence des dédoublements de raies R.P.E. pour des directions particulières du champ magnétique par rapport aux axes cristallographiques, l'analyse de ces dédoublements et l'étude de l'élargissement des raies optiques, nous permettant de mesurer la désorientation à l'intérieur du cristal.

1. Technique expérimentale. - Les cristaux de rubis étudiés nous ont été fournis par Ugine Kuhlmann et se présentent à l'origine comme des parallélépipèdes de $5 \times 2 \times 2 \mathrm{~mm}^{3}$. Leur concentration en ion chrome est de $0,1 \mathrm{Cr} \%$.

1.1 Déformations PlastiQues. - Les principaux systèmes de glissement du corindon sont les glissements basal, prismatique et pyramidal. Les conditions de déformation sont celles indiquées dans de précédents articles [16, 17]. Le glissement basal correspond

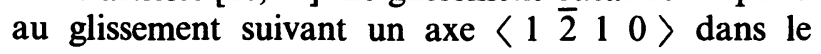
plan $\left(\begin{array}{llll}0 & 0 & 0 & 1\end{array}\right)$ et le glissement prismatique a un glissement suivant la direction $\left\langle\begin{array}{llllll}1 & 0 & \overline{1} & 0\end{array}\right\rangle$ dans le plan $\left(\begin{array}{llll}1 & \overline{2} & 1 & 0\end{array}\right)$ (Fig. 1). C'est par rapport à l'axe $\left\langle\begin{array}{llll}0 & 0 & 0 & 1\end{array}\right\rangle$ nommé c qu'est repérée la direction du champ magnétique $\mathbf{H}$ lors des expériences de R.P.E.

Le glissement basal des cristaux de rubis a été activé à $1400^{\circ} \mathrm{C}$ avec une vitesse de déformation $\dot{\varepsilon}=1,66 \times 10^{-4} \mathrm{~s}^{-1}$ (taux de déformation $8 \%$ ). La figure 2 représente le plan basal et la direction de la contrainte. 


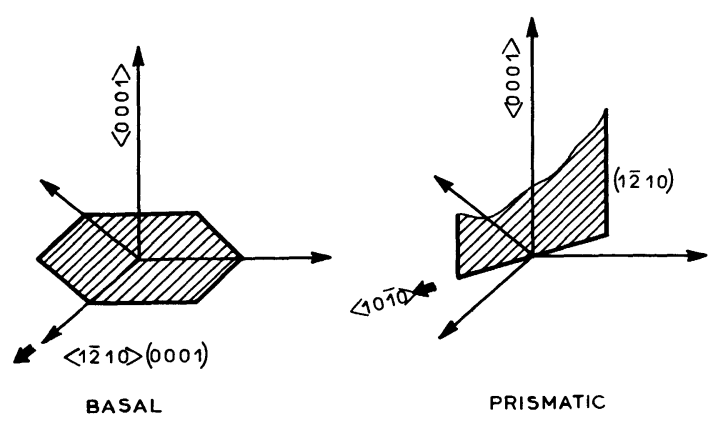

Fig. 1. - Représentation schématique des différents systèmes de glissement du corindon d'après [16].

[Basal and prismatic glide system of corundum from [16].]

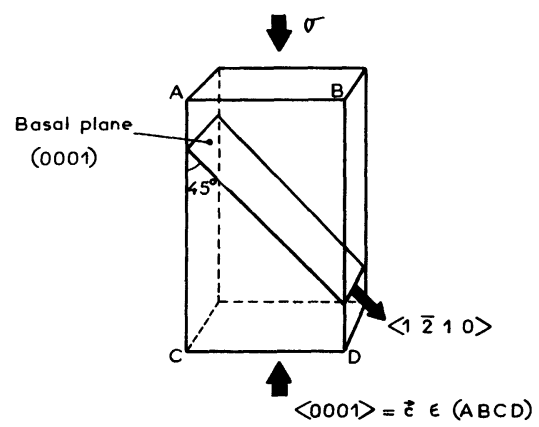

Fig. 2. - Direction de la contrainte $\sigma$ et du glissement basal.

[Stress direction $\sigma$ and basal plane.]

Le glissement prismatique a été activé à $1500^{\circ} \mathrm{C}$ avec la même vitesse $\dot{\varepsilon}=1,66 \times 10^{-4} \mathrm{~s}^{-1}$ (taux de déformation de 8/1 000).

1.2 SPeCtroscopie R.P.E. - Les cristaux de rubis déformés plastiquement ont été étudiés en R.P.E. L'ion $\mathrm{Cr}^{3+}$ dans le corindon est soumis à un champ de symétrie trigonale. Ce champ cristallin et le couplage spin-orbite lèvent la dégénérescence du niveau fondamental ${ }^{4} \mathrm{~A}_{2}$ en 2 sous-niveaux correspondant à $m_{s}= \pm \frac{3}{2}$ et $m_{s}= \pm \frac{1}{2}$ séparés par $0,38 \mathrm{~cm}^{-1}[1-4]$. Le champ magnétique externe $H$ lève la dégénérescence entre les sous-niveaux $m_{s}=+\frac{3}{2}, m_{s}=-\frac{3}{2}$ et $m_{s}=+\frac{1}{2}, m_{s}=-\frac{1}{2}$ entre lesquels une onde hyperfréquence à $9,2 \mathrm{GHz}\left(0,31 \mathrm{~cm}^{-1}\right)$ induit des transitions (Fig. 3). En pratique, l'onde hyperfréquence reste donc fixe et l'on fait varier le champ magnétique $\mathbf{H}$ pour observer les transitions. En prenant l'axe $\left.\mathbf{c}<\begin{array}{llll}0 & 0 & 0 & 1\end{array}\right\rangle$ comme axe de quantification, l'hamiltonien de spin s'écrit [1] :

$$
\begin{aligned}
\mathscr{H}=g_{\|} \beta H_{z} S_{z}+g_{\perp} \beta\left(H_{x} S_{x}\right. & \left.+H_{y} S_{y}\right)+ \\
& +D\left(S_{z}^{2}-S(S+1)\right)
\end{aligned}
$$

avec

$$
\begin{aligned}
g_{\|} & =1,9894 \pm 0,0006 \\
g_{\perp} & =1,9867 \pm 0,0006 \\
2 D & =-0,38 \mathrm{~cm}^{-1} .
\end{aligned}
$$

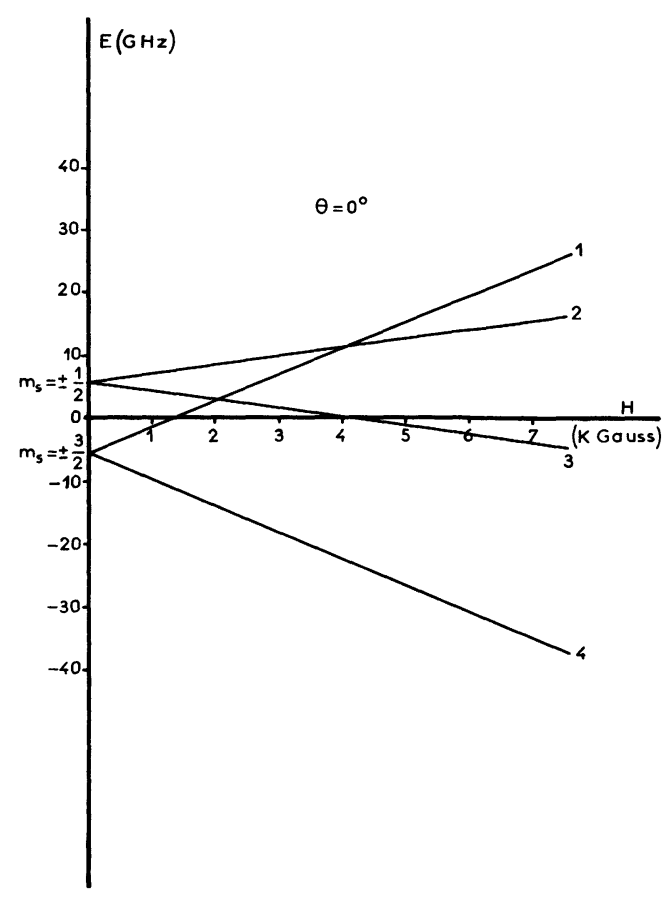

Fig. 3. - Séparation des niveaux $m_{s}= \pm \frac{3}{2}$ et $m_{s}= \pm \frac{1}{2}$. du niveau fondamental ${ }^{4} \mathrm{~A}_{2}$ de $\mathrm{Cr}^{3+}$ par un champ magnétique $\mathrm{H}$ parallèle à l'axe c $\left(\theta=0^{\circ}\right)$.

[Splitting of the $m_{s}= \pm \frac{3}{2}$ and $m_{s}= \pm \frac{1}{2}$ sublevels of ${ }^{4} \mathrm{~A}_{2}$ ground state of $\mathrm{Cr}^{3+}$ for an applied magnetic field $\mathrm{H}$ parallel to the $\mathrm{c}$ axis $\left(\theta=0^{\circ}\right)$.]

Pour un cristal non déformé lorsqu'on fait varier l'angle $\theta$, angle entre la direction du champ magnétique $\mathbf{H}$ et l'axe optique $\mathbf{c}$, on obtient un réseau de courbes $\theta=f(H)$ représenté en traits pleins sur la figure 4. Les niveaux sont numérotés 1, 2, 3, 4(Fig. 3) le niveau supérieur portant toujours le numéro 1 . Les expressions " champ fort " (H.F.) et " champ faible " (L.F.) permettent de distinguer lorsque plusieurs transitions portent le même nom [3].

Dans le cas de cristaux non déformés, ces courbes $\theta=f(H)$ sont semblables à celles obtenues précédemment par différents auteurs $[3,4]$ et sont en bon accord avec celles calculées par Schulz du Bois [18].

Dans le cas de cristaux déformés plastiquement et ayant subi un glissement basal, les spectres R.P.E. réalisés avec $H$ contenu dans le plan $\left(\begin{array}{llll}1 & 0 & \overline{1} & 0\end{array}\right)$ et ceux avec $\mathbf{H}$ contenu dans le plan $\left(\begin{array}{llll}1 & \overline{2} & 1 & 0\end{array}\right)$ sont différents :

- Lorsque $\mathbf{H}$ est contenu dans le plan $\left(\begin{array}{llll}1 & 0 & \overline{1} & 0\end{array}\right)$ les raies de résonance se dédoublent pour certaines valeurs de $\theta$ (Fig. 5). Chacune des raies a un dédoublement qui lui est propre en fonction de cet angle. Les composantes dédoublées des raies de résonance sont indiquées en tirets sur la figure 4. On remarque que les écarts entre les 2 composantes d'une raie sont inversement proportionnels à la pente de la courbe $\theta=f(H)$ pour la raie considérée. Par exemple, la raie 2-3 champ faible n'est pratiquement pas scindée dans le cristal déformé, la pente correspondante dans 


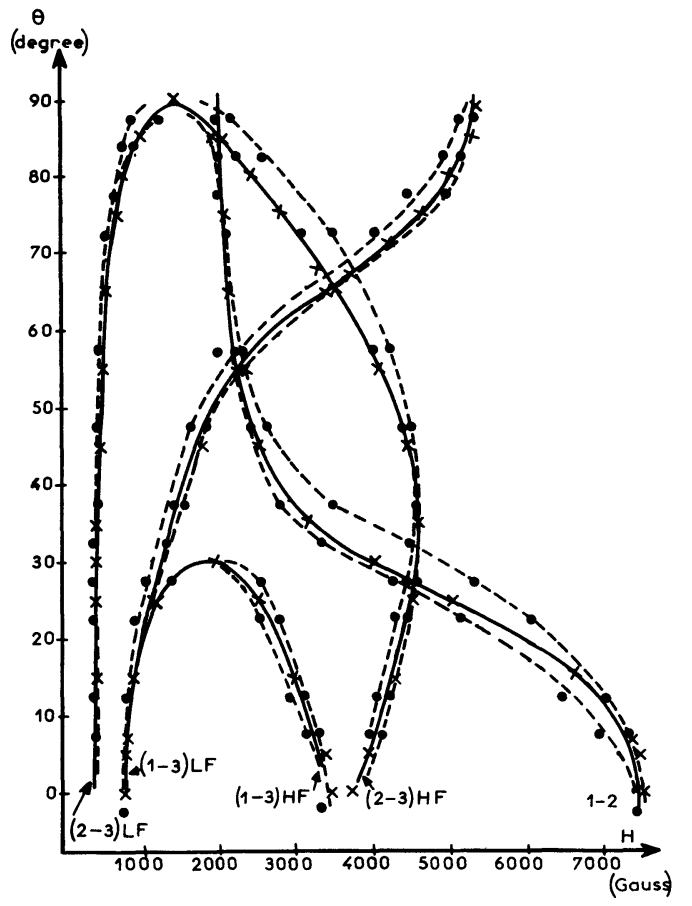

Fig. 4. - Positions expérimentales des raies de R.P.E. (onde hyperfréquence à $9,2 \mathrm{GHz}$ ) pour différentes orientations $\theta$ entre le champ magnétique $\mathbf{H}$ et l'axe optique $\mathbf{c}$ : cristal non déformé; - glissement basal $\mathbf{H}$ contenu dans $\left(\begin{array}{llll}1 & 0 & 1 & 0\end{array}\right) ; \times$ glissement basal $\mathbf{H}$ contenu dans $\left(\begin{array}{llll}1 & \overline{2} & 1 & 0\end{array}\right)$.

[Experimental plot of E.P.R. lines at $9.2 \mathrm{GHz}$ for different $\theta$ between the magnetic field $H$ and the optical axis $c$ : non deformed crystal; $\odot$ basal glide, $\mathbf{H}$ being in the $\left(\begin{array}{llll}1 & 0 & \overline{1} & 0\end{array}\right)$ plane; $\times$ basal glide, $\mathbf{H}$ being in the $\left(\begin{array}{llll}1 & \overline{2} & 1 & 0\end{array}\right)$ plane.]

le diagramme $\theta=f(H)$ étant pratiquement infinie alors que c'est dans la région de pente minimale que la raie 1-2 se dédouble le plus largement.

- Lorsque la direction du champ magnétique $\mathbf{H}$ est contenue dans le plan $\left(\begin{array}{llll}1 & \overline{2} & 1 & 0\end{array}\right)$ il n'y a pas de séparation des raies de résonance. Sur la figure 4, la position d'une raie est dans ce cas indiquée par une croix $(x)$ et suit très bien la courbe correspondante du cristal non déformé (en trait plein).

1.3 SPECTROSCOPIE OPTIQUE. - Les raies d'émission du rubis dans le visible correspondent aux transitions ${ }^{2} E \rightarrow{ }^{4} A_{2}$ (émissions $R_{1}$ et $R_{2}$ ) et à des émissions d'ions chrome voisins couplés par l'interaction d'échange (raies « de paire » $N_{1}$ et $N_{2}$ principalement, correspondant respectivement aux ions $\mathrm{Cr}^{3+}$ en position de troisième plus proche voisin et quatrième plus proche voisin). L'influence des contraintes élastiques a été étudiée tant sur les raies correspondant aux ions isolés (raies $R_{1}$ et $R_{2}$ [10]) que sur les niveaux de paires $N_{1}$ et $N_{2}[19,20]$. Les raies $R_{1}$ et $R_{2}$ subissent un simple déplacement sous l'effet des contraintes parallèles ou perpendiculaires à l'axe optique [10] alors que les raies de paires peuvent être scindées en deux par l'application de contraintes

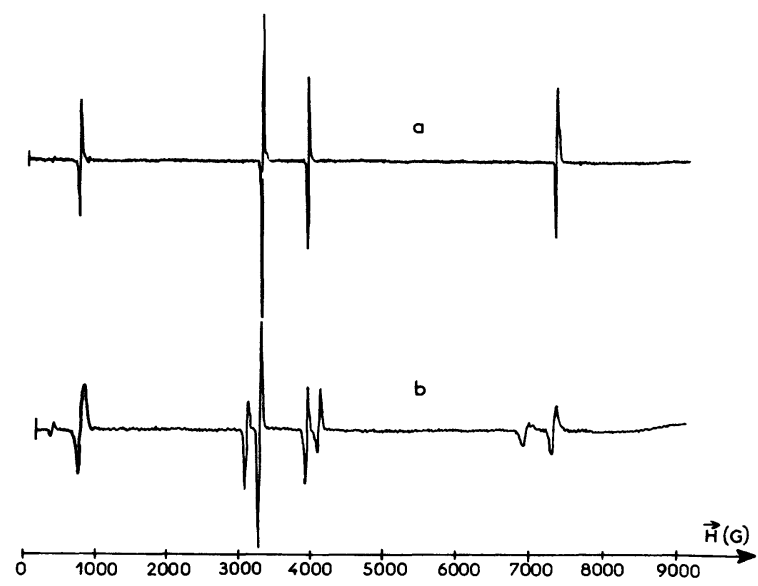

Fig. 5. - Spectres R.P.E. lorsque le champ magnétique $\mathbf{H}$ est contenu dans le plan $\left(\begin{array}{llll}1 & 0 & \overline{1} & 0\end{array}\right)$ et fait un angle $\theta=5^{\circ}$ avec l'axe optique : $a$ ) cristal non déformé ; $b$ ) cristal déformé (glissement basal).

[E.P.R. spectrum, the magnetic field $\mathbf{H}$ being in the $\left(\begin{array}{llll}1 & 0 & \overline{1} & 0\end{array}\right)$ plane and making the angle $\theta=5^{\circ}$ with the optical axis : $a$ ) non-deformed crystal; $b$ ) deformed crystal (basal glide).]

suffisamment élevées. Expérimentalement, il est donc plus aisé d'étudier les raies de paires qui s'élargissent rapidement sous l'action de la contrainte que les raies $\mathrm{R}$ dont les déplacements sont plus difficiles à mesurer. Nous avons donc déterminé les contraintes dues aux déformations plastiques sur la raie $\mathbf{N}_{2}$ des cristaux. Ceci peut être fait en comparant les largeurs des raies $\mathbf{N}_{2}$ pour des cristaux non déformés, déformés ayant subi un glissement basal ou déformés ayant subi un glissement prismatique. La figure 6 permet la

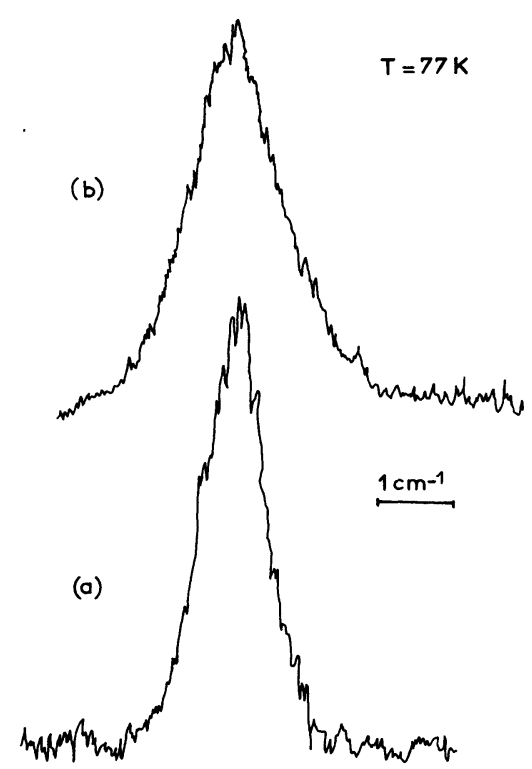

Fig. 6. - Largeur de la raie d'émission $\mathrm{N}_{2}$ de $\mathrm{Cr}^{3+}-\alpha \mathrm{Al}_{2} \mathrm{O}_{3}$ : a) cristal non déformé; $b$ ) cristal ayant subi un glissement basal.

$\left[\mathrm{N}_{2}\right.$ emission line in $\left.\mathrm{Cr}^{3+}-\alpha \mathrm{Al}_{2} \mathrm{O}_{3}: a\right)$ non deformed crystal; $b$ ) deformed crystal along the basal glide.] 
comparaison de la largeur de la raie $\mathrm{N}_{2}$ pour un cristal non déformé et un cristal déformé (glissement basal) à $77 \mathrm{~K}$. Les raies $R_{1}, R_{2}, N_{1}$ et $N_{2}$ du cristal déformé ayant subi un glissement pyramidal s'élargissent également.

2. Interprétation et discussion. - Les résultats des mesures R.P.E. font apparaître une différence de comportement suivant la direction du champ magnétique $\mathbf{H}$ par rapport aux axes cristallographiques. La figure 4 montre que lorsque $\mathbf{H}$ est contenu dans le plan $\left(\begin{array}{llll}1 & 0 & \overline{1} & 0\end{array}\right)$ chacune des raies de résonance est dédoublée; cette séparation varie de manière inversement proportionnelle à la pente de la courbe $\theta=f(H)$. La transition 1-2 est celle où apparaît le plus fort dédoublement puisque pour $\theta=25^{\circ}$ on obtient une séparation des raies R.P.E. de l'ordre de 1000 gauss alors que la transition 2-3 champ faible est élargie mais n'apparaît scindée qu'au voisinage de $\theta=90^{\circ}$.

Deux catégories d'hypothèses peuvent être envisagées pour décrire ces effets. D'une manière analogue à ce qui a été envisagé pour étudier l'élargissement inhomogène des raies R.P.E. d'un cristal de rubis $[21,22]$ on peut soit considérer que l'échantillon après déformation est un ensemble de blocs désorientés, c'est ce que nous appelons l'effet de désorientation, soit considérer que les effets obtenus sont dus à des contraintes internes. Dans le premier cas, le site de chacun des ions n'est pas perturbé mais différentes régions du cristal ont des axes c qui ne sont pas parallèles entre eux; chacune de ces régions est supposée parfaite mais elles sont désorientées entre elles. Au contraire, dans un modèle où l'on fait intervenir les contraintes internes, le site de chacun des ions chrome est perturbé; les ions oxygène autour de l'ion $\mathrm{Cr}^{3+}$ sont déplacés par la contrainte. Du point de vue R.P.E. l'effet de contraintes internes peut être décrit [23] en ajoutant à l'hamiltonien de spin (1) un terme

$$
\mathcal{H}^{\prime}=\sum_{i j} D_{i j} S_{i} S_{j}
$$

Le tenseur $D_{i j}$ étant relié aux contraintes $e_{k l}$ par [24] :

$$
D_{i j}=\sum_{i j k l} G_{i j k l} e_{k l}
$$

E. Feher [25] a déduit de ces expressions le déplacement du champ magnétique auquel apparaît une transition pour une direction de contrainte et de champ magnétique quelconque, les angles $\theta^{\prime}$ et $\varphi^{\prime}$ étant les angles polaires entre la direction du champ magnétique et les axes 1, 2, 3 de la déformation :

$$
\begin{aligned}
\delta H_{m_{s} \rightarrow m_{s-1}} & =-\left(2 m_{s}-1\right) \times \\
& \times\left[D\left(\frac{3}{2} \cos ^{2} \theta^{\prime}-\frac{1}{2}\right)+\frac{3}{2} E \cos ^{2} \varphi^{\prime} \sin ^{2} \theta^{\prime}\right]
\end{aligned}
$$

$\operatorname{avec} D=\frac{3}{2} D_{33}$ et $E=\frac{1}{2}\left(D_{11}-D_{22}\right)$.
Une telle expression montre qu'au premier ordre la transition $\frac{1}{2} \rightarrow-\frac{1}{2}$ (1-3 champ fort) n'est pas scindée par les contraintes internes $\left(2 m_{s}-1=0\right)$ ce qui devrait nous permettre de conclure sur le modèle à considérer pour expliquer nos résultats expérimentaux. Cependant, R. F. Wenzel et Y. W. Kim [26] ont montré qu'en ce qui concerne l'élargissement des raies R.P.E. lorsqu'on considère les effets d'ordre plus élevé pour les contraintes, il n'est plus possible de conclure sur un des effets plutôt que l'autre. La figure 3 de l'article de Wenzel et Kim montre que pour toutes les raies observées les 2 modèles, contraintes ou désorientation, ont des effets semblables et rendent compte aussi bien l'un que l'autre des résultats expérimentaux.

Les mesures R.P.E. ne permettent pas de choisir entre les deux hypothèses. Considérons les résultats donnés par les mesures optiques : la raie $\mathrm{N}_{2}$ du rubis (Fig. 6) est environ 1,5 fois plus large dans le cristal déformé (glissement basal) que dans le cristal non déformé $\left(\Delta v=1,5 \mathrm{~cm}^{-1}\right.$ contre $\Delta v=1 \mathrm{~cm}^{-1}$ à 77 K). A. A. Kaplyanskii et A. K. Przhevuskii [20] ont observé qu'une contrainte élastique de $100 \mathrm{~kg} . \mathrm{mm}^{-2}\left(10^{9} \mathrm{~N} . \mathrm{m}^{-2}\right)$ perpendiculaire à l'axe optique c produit une séparation maximum des raies de $6,2 \mathrm{~cm}^{-1}$. Par comparaison l'élargissement observé dans nos expériences nous amène à penser que la raie $\mathrm{N}_{2}$ est soumise à une contrainte maximum environ 10 fois plus faible $\left(10^{8} \mathrm{~N} \cdot \mathrm{m}^{-2}\right)$. Ces mesures optiques nous donnent l'ordre de grandeur de la contrainte interne des cristaux étudiés. Il est donc possible de comparer nos résultats expérimentaux en R.P.E. avec l'effet prévu de ces contraintes. Les résultats de Hemphill et al. [11] montrent que suivant la direction de la contrainte appliquée, on peut en R.P.E. observer soit un simple déplacement soit un clivage des raies du rubis. L'ordre de grandeur maximum des déplacements observés dans ces expériences est d'environ 10 gauss pour une contrainte de $5 \times 10^{7} \mathrm{~N} . \mathrm{m}^{-2}$ [10]. Pour la contrainte interne estimée dans nos échantillons à partir des résultats de spectroscopie optique, les effets doivent alors être de l'ordre de 20 gauss au maximum alors que nos résultats expérimentaux (Fig. 4) montrent des effets beaucoup plus importants. La raie 2-3 (champ fort) par exemple est clivée par une contrainte interne à $45^{\circ}$ de l'axe c. La séparation des 2 composantes, qui varie peu avec l'orientation, peut être estimée à 10 gauss pour une contrainte de $10^{8} \mathrm{~N} \cdot \mathrm{m}^{-2}[10,11]$ alors que nos mesures montrent que les 2 composantes de cette raie 2-3 sont pour certaines directions séparées par 300 gauss (Fig. 4). L'hypothèse des contraintes internes pour expliquer les dédoublements R.P.E. observés doit donc être écartée.

Dans l'hypothèse où il y a désorientation, à la suite d'un glissement basal, les différents blocs désorientés dont les axes $c$ font entre eux un angle $\Delta \theta$ ont des raies de résonance séparées par un champ magnétique $\Delta H$. A partir de la mesure de $\Delta H$ pour différents 
angles $\theta$ il est possible de déterminer la désorientation $\Delta \theta$ au moyen de

$$
\Delta \theta=\frac{\mathrm{d} \theta}{\mathrm{d} H} \Delta H .
$$

Lorsque le champ magnétique est contenu dans le plan (1 $0 \overline{1}$ 0) on a pu mesurer expérimentalement $\Delta H$ pour diverses valeurs de $\theta$ et donc l'expression ci-contre permet de déterminer (Tableau I)

$$
\Delta \theta=6,2^{\circ} \pm 1,5^{\circ} \text {. }
$$

Ces mesures ont été effectuées sur la raie 1-2 entre 7,50 et $47,5^{\circ}$ car c'est pour cette transition et pour ces valeurs de $\theta$ que la sensibilité est la meilleure, le terme $\mathrm{d} \theta / \mathrm{d} H$ étant maximum. Les résultats obtenus sur d'autres raies sont cohérents avec la mesure ci-dessus.

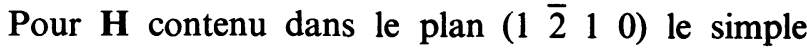
élargissement des raies, sans dédoublement, montre que la désorientation est beaucoup plus faible. Nous mettons donc ici en évidence, pour des cristaux ayant subi un glissement basal, une désorientation de l'axe c de l'ordre de $6^{\circ}$ dans le plan $\left(\begin{array}{llll}1 & 0 & \overline{1} & 0\end{array}\right)$. Cette désorientation peut être attribuée soit à des sousjoints de flexion provenant du regroupement de dislocations le long d'une surface, l'axe de rotation des sous-joints étant la direction $\left\langle\begin{array}{llll}1 & 0 & \overline{1} & 0\end{array}\right\rangle$, soit à une déformation hétérogène avec rotation de zones du cristal par rapport à d'autres, l'axe de rotation étant aussi l'axe $\left\langle\begin{array}{llll}1 & 0 & \overline{1} & 0\end{array}\right\rangle$. Les effets d'une telle déformation hétérogène du cristal, combinaison de cisaillements et de rotations, sont décrits en détail par Castaing et al. [27]. Cette hypothèse paraît plus plausible que celle des sous-joints si l'on compare les intensités des raies R.P.E. : lorsqu'une raie donnée est scindée en deux, l'intensité des 2 composantes est du même ordre de grandeur ce qui signifie que les sites d'ions $\mathrm{Cr}^{3+}$ se partagent à peu près également en 2 catégories faisant entre elles l'angle $\Delta \theta$. Le cas de sousjoints ferait intervenir un pourcentage d'ions $\mathrm{Cr}^{3+}$ beaucoup plus faible par rapport au nombre total d'ions $\mathrm{Cr}^{3+}$ ce qui se traduirait par une intensité beaucoup plus faible de la raie R.P.E. correspondant aux sites désorientés. Nous pouvons donc conclure que l'effet observé est dû à la déformation hétérogène du cristal avec rotations autour d'un axe $\left\langle\begin{array}{llll}1 & 0 & 1 & 0\end{array}\right\rangle$.

Pour les cristaux, où un glissement prismatique a été activé, aucune direction du champ magnétique $\mathbf{H}$ en R.P.E. ne met en évidence de désorientation de l'axe c. Ce résultat paraît conforme à ce que l'on peut prévoir d'une part à cause du faible taux de déformation de l'échantillon ayant subi le glissement prismatique et d'autre part, d'après [27], lorsqu'il existe une déformation hétérogène celle-ci est plus importante pour une direction d'observation parallèle à l'axe $\left\langle\begin{array}{llll}0 & 0 & 0 & 1\end{array}\right)$. Les axes $\mathbf{c}$ restent alors parallèles entre eux : la R.P.E. qui n'est sensible qu'à la rotation de l'axe c ne permet donc pas de mettre en évidence un effet. Ceci explique que dans le cas d'un glissement prismatique même si une déformation hétérogène du cristal est activée, il n'existe pas d'effet sur le spectre R.P.E.

En conclusion, le recoupement de résultats obtenus par spectroscopie optique et par R.P.E. est un moyen intéressant d'analyser les cristaux soumis à des déformations plastiques puisque dans le cas où ces déformations produisent des rotations de l'axe c dans différentes régions du cristal, il est possible de mesurer la désorientation correspondante. L'application de telles techniques de caractérisation microscopique de la déformation plastique peut être envisagée pour d'autres matériaux cristallins isolants ou semi-conducteurs dans lesquels peuvent être incorporés des ions donnant des spectres R.P.E. ou optiques sensibles aux désorientations ou aux contraintes internes. Du point de vue optique l'existence de raies fines d'absorption ou de fluorescence est nécessaire pour observer l'effet des contraintes internes. Des ions comme $\mathrm{Cr}^{3+}$ ou $\mathrm{V}^{2+}$ sont bien appropriés. Dans le cas où la symétrie est plus élevée, par exemple Oh comme dans $\mathrm{MgO}$ au lieu de $\mathrm{D}_{3 \mathrm{~d}}$ comme dans l'alumine, des contraintes internes se transformant comme les fonctions de base de la représentation irréductible e (tétragonales par exemple) scindent les raies de fluorescence des ions isolés en deux. Dans ce cas, une caractérisation des contraintes internes peut être plus directe. Les nou-

Tableau I. - Désorientation $\Delta \theta$ mesurée à partir du dédoublement $\Delta H$ de la transition 1-2 pour un cristal déformé

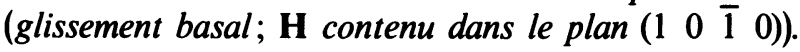

[Disorientation $\Delta \theta$ measured from the $\Delta H$ splitting of the 1-2 transition for a deformed crystal (basal glide; $\mathbf{H}$ being in the $\left(\begin{array}{lllll}1 & 0 & \overline{1} & 0\end{array}\right)$ plane).]

\begin{tabular}{|c|c|c|c|c|c|c|c|}
\cline { 2 - 7 } \multicolumn{1}{c|}{} & $7^{\circ} 5$ & $12^{\circ} 5$ & $22^{\circ} 5$ & $27^{\circ} 5$ & $32^{\circ} 5$ & $37^{\circ} 5$ & $47^{\circ} 5$ \\
\hline$\Delta H(\mathrm{G})$ & 380 & 562 & 912 & 1050 & 1100 & 675 & 212 \\
\hline$\frac{\mathrm{d} \theta}{\mathrm{d} H} \times 10^{3}$ (rad./G) & 0,29 & 0,17 & 0,10 & 0,08 & 0,11 & 0,19 & 0,63 \\
\hline$\Delta \theta$ & $6^{\circ} 3$ & $5^{\circ} 5$ & $5^{\circ} 2$ & $4^{\circ} 8$ & $6^{\circ} 9$ & $7^{\circ} 3$ & $7^{\circ} 6$ \\
\hline
\end{tabular}


velles techniques d'affinement des raies de fluorescence par excitation laser (F.L.N.) peuvent de plus permettre une évaluation précise de ces contraintes [28].
Remerciements. - Nous remercions J. Castaing pour la lecture critique de ce manuscrit et ses suggestions pertinentes.

\section{Bibliographie}

[1] Manenkov, A. A. et Prokhorov, A. M., Sov. Phys. JETP 1 (1955) 611.

[2] Zaripov, M. M. et Shamonin, I. I., Sov. Phys. JETP 3 (1956) 171.

[3] Duclaux, A. M., Ann. Phys. 3 (1968) 89.

[4] Geusic, J. E., Phys. Rev. 102 (1956) 1252.

[5] Margerie, J., Thèse de Doctorat d'Etat, Paris (1965).

[6] McClure, D. S., J. Chem. Phys. 36 (1962) 2757.

[7] Lacroix, R., Duval, E., Champagnon, B. et Louat, R., Phys. Status Solidi (b) 68 (1975) 473.

[8] Louat, R., Lacroix, R., Duval, E. et Champagnon, B., Phys. Status Solidi (b) 69 (1975) 33.

[9] Champagnon, B. et Duval, E., J. Phys. C. 13 (1980) 131.

[10] Feher, E. et Sturge, M. D.. Phys. Rev. 172A (1968) 244.

[11] Hemphill, R. B., Donoho, P. L. et MacDonald, E. D., Phys. Rev. 146 (1966) 329.

[12] Stauss, G. H. et Krebs, J. J., Phys. Rev. B 22 (1980) 2050.

[13] Gehlhoff, W. et Segsa, K. H., Phys. Status Solidi (b) (1981) 171.

[14] Wosinski, T., Crys. Research and Technology 16 (1981) 217.
[15] Wosinski, T., Phys. Status Solidi (a) 60 (1980) K149.

[16] Cadoz, J., Thèse de Doctorat d'Etat, Orsay (1978).

[17] Cadoz, J., Castaing, J. et Philibert, J., Revue Phys. Appl. 16 (1981) 135.

[18] Shulz Du Bois, E. O., Bell. Syst. Techn. J. 38 (1959) 271.

[19] Kaplyanskiı, A. A. et Przhevuskij, A. K., Sov. Phys. Dockl. 7 (1962) 37.

[20] Kaplyanskil, A. A. et Przhevuskit, A. K., Sov. Phys. Solid State 9 (1967) 190.

[21] Curtis, D. J., KiRKBY, C. J. et ThORP, J. S., Brit. J. Appl. Phys. 16 (1965) 1681

[22] Shaltiel, D. et Low, W., Phys. Rev. 124 (1961) 1062.

[23] Watkins, G. D. et Feher, E., Bull. Am. Phys. Soc. 7 (1962) 29.

[24] Donoho, P. L., Phys. Rev. 133A (1964) 1080.

[25] Feher, E., Phys. Rev. 136A (1964) 145.

[26] Wenzel, R. F. et KIM, Y. M., Phys. Rev. 140A (1965) 1592.

[27] Castaing, J., Cadoz, J. et Kirby, S. H., J. Am. Ceram. Soc. 64 (1981) 504.

[28] Rabia, M. K., Boyrivent, A. et Duval, E., J. Physique Lett. (à paraître). 